\title{
Controlling transport in triphenylene-based metal-organic frameworks
}

\author{
Grigorii Skorupskii, ${ }^{a}$ Benjamin A. Trump, ${ }^{b}$ Thomas W. Kaselc, Craig M. Brown, ${ }^{b, d}$ \\ Christopher H. Hendon, ${ }^{\mathrm{c}}$ Mircea Dincăa
}

aDepartment of Chemistry, Massachusetts Institute of Technology, 77 Massachusetts Avenue, Cambridge, Massachusetts 02139, United States, gsk@mit.edu, mdinca@mit.edu ${ }^{b}$ Center for Neutron Research, National Institute of Standards and Technology, Gaithersburg, Maryland 20899, United States, benjamin.trump@nist.gov

'Materials Science Institute, Department of Chemistry and Biochemistry, University of Oregon, Eugene, Oregon 97403, United States, chendon@uoregon.edu

dDepartment of Chemical and Biomolecular Engineering, University of Delaware, Newark,

Delaware 19716, United States, craig.brown@nist.gov

Metal-organic frameworks (MOFs) show much promise as a flexible platform for applied materials thanks to their intrinsic tunability and unique porosity at the nanoscale.[1] Of particular interest are two-dimensional $\pi$-conjugated MOFs based on hexa-substituted triphenylenes[2] and benzenes,[3] where high electrical conductivities led to applications in supercapacitors,[4] thermoelectrics,[5] and electrochemical catalysts.[6] The vast majority of such MOFs, however, show poor crystallinity which prevents detailed structure-function relationship studies.

We report a new series of well-ordered and highly conductive triphenylene-based MOF materials, and show how their transport and optical properties can be controlled through fine tuning of the structure. Pressed pellets of the materials show electrical conductivity values of up to $0.05 \mathrm{~S} / \mathrm{cm}$. Variable temperature conductivity measurements reveal semiconducting behavior. Powder X-ray diffraction and density functional theory provide insight into the origin of the transport properties.

\section{$\underline{\text { References }}$}

[1] Ko, M., Mendecki, L. \& Mirica, K. A. (2018). Chem. Commun. 54, 7873-7891.

[2] Sheberla, D. et al. (2014). J. Am. Chem. Soc. 136, 8859-8862.

[3] Dou, J.-H. et al. (2017). J. Am. Chem. Soc. 139, 13608-13611.

[4] Sheberla, D. et al. (2016). Nat. Mater. 16, 220-224.

[5] Sun, L. et al. (2017). Joule 1, 168-177.

[6] Miner, E. M. et al. (2016). Nat. Commun. 7, 10942. 